\title{
Strong dispersive effects in the light-scattering mean free path in photonic gaps
}

\author{
P. D. García, R. Sapienza, L. S. Froufe-Pérez, and C. López \\ Instituto de Ciencia de Materiales de Madrid (CSIC) and Unidad Asociada CSIC-UVigo, \\ Cantoblanco, 28049 Madrid, Spain \\ luis.froufe@icmm.csic.es
}

Light propagates along straight lines from the luminous body to the body illuminated and bends or breaks those lines (scatters) when passes out of one medium into another ${ }^{[1]}$. Contemporary photonic science goes one step further and provides materials, like photonic crystals, in which light scattering is controlled through interference from the bulk nanostructure instead of the system boundaries. Photonic crystals modify the vacuum modes, change the available phase space for light propagation opening photonic gaps which inhibits some (or even all) directions, while increase the density of light states around the band-gap frequencies. We present here ${ }^{[2]}$ direct evidence of one order of magnitude variance in the scattering mean free path in photonic crystals for just $\sim 25 \mathrm{~nm}$ wavelength shift around the band-gap. This dramatic and resonant dispersion is unique and comes from the light interference rather than from material properties. The co-existence of periodicity (the crystal) and disorder (structural imperfections) allows controlling light scattering and diffusion. In particular, light scattering enhancement at band-edge mode has been predicted to be the key for achieving Anderson localization of light. Relying on very high quality photonic crystals, with losses of $\sim 100$ $\mathrm{dB} / \mathrm{cm}$, we confirm that scattered photons dramatically feel the density of states when propagating though photonic crystals. We present a method that permits an accurate measurement of the light scattering mean free path and a precise assessment of the quality of photonic materials. A control of light diffusion in ordered dielectrics is an unexplored route to photonic devices based on disorder rather than order, in analogy to the majority of microelectronic devices that are based on the control of electron diffusion in atomic crystals ${ }^{[3]}$.

We show ${ }^{[2]}$ that a controlled smooth transition from ballistic to diffuse transport in photonic crystals can be induced by the introduction of extrinsic disorder. We find that the strength of scattering is strongly determined by the spectral function, which induces immense, up to 20fold, variations in the scattering mean free path. We propose the scattering mean free path as a robust, easy to measure, figure of merit in assessing the quality of photonic crystals for technological applications. The possibility of controlling light scattering and diffusion in nanostructured optical media has important implications not only in testing the quality of photonic devices but also in properly addressing the proximity to the onset of Anderson in disordered lattices or for the spectral control of lasing emission from disordered/ordered active media $^{[4]}$.

\section{References:}

[1] I. Newton, A treatise of the reflections, refractions, inflections and colours of light (Opticks) (London, 1704; reprinted by Dover, New York, 1952).

[2] P. D. García, R. Sapienza, L. S. Froufe-Pérez, and C. López, Phys. Rev. B 79 (2009) 241109R.

[3] C. Kittel, Introdution to Solid State Physics (J. Whyley and sons, N. York, 1971).

[4] D. S. Wiersma,, Nature Phys. 4 (2008) 359; S. Gottardo, R. Sapienza, P.D. García, A. Blanco, D. S. Wiersma, and C. López, Nature Phot. 2 (2008) 429. 